International Journal of Pure and Applied Mathematics

Volume 116 No. 1 2017, 169-175

ISSN: 1311-8080 (printed version); ISSN: 1314-3395 (on-line version)

url: http://www.ijpam.eu

doi: 10.12732/ijpam.v116i1.17

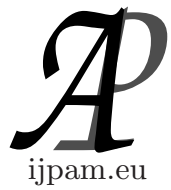

\title{
STRONGLY PRIME RADICALS OF A TERNARY SEMIGROUP
}

\author{
Mani P. Seetha ${ }^{1}$, Y. Sarala ${ }^{2}$, A. Anjaneyulu ${ }^{3}$, P. Bindu ${ }^{4}$ \\ 1,3,4 Department of Mathematics \\ K.L. University \\ Guntur Dt., A.P., INDIA \\ ${ }^{2}$ Department of Mathematics \\ National Institute of Technology \\ A.P., INDIA
}

\begin{abstract}
In this article we introduce the notion of right stronglyprime radical of a ternarysemigroup and study it. We also introduce the notion of a super sp-system of a ternarysemigroup and characterize it.
\end{abstract}

AMS Subject Classification: 20M12, 20M17

Key Words: right stronglyprime ternarysemigroup, right stronglyprime ideal, super spsystem, right stronglyprime radical

\section{Introduction}

The literature of the theory of ternary operations is vast and scatter over diverse areas of mathematics. In 1932 Lehmer[6] introduced the concept of ternary algebraic systems. The algebraic system called triplexes was investigated by Lehmer which turn out to be commutative ternary groups. Kasner[3] also studied the structures and give the ideal of $n$-ary algebras. Ternarysemigroups are universal algebras with one associative ternary operation. The ideal theory in ternary-

Received: July 14, 2017

Revised: $\quad$ August 6, 2017

Published: August 30, 2017

$\S$ Correspondence author (c) 2017 Academic Publications, Ltd. url: www.acadpubl.eu 
semigroup was introduced by Sioson [8]. Shabir and Bashir[9] launched prime ideals in ternarysemigroups. The class of stronglyprime semigroups is an interesting class of semigroups because it has some interesting properties which are similar to the properties of commutation domains. Some earlier works of ternarysemigroup may be found in $[1,2,4,5,7]$. Throughout this paper $T$ will always denote a ternarysemigroup with zero and $T^{*}=T \backslash\{0\}$. In this paper we introduce and study the notion of right stronglyprime radical in ternarysemigroup.

\section{Right Strongly Prime Radicals of a TernarySemigroup}

Definition 2.1. A ternarysemigroup $T$ is called a right stronglyprime if for every $x \in T^{*}$ there exist finite subsets $F_{1}, F_{2}, F_{3}$ of $T$ depending on $x$ such that $x F_{1} F_{2} F_{3} y=\{0\} \Rightarrow y=0$ for all $y \in T$.

Example 2.2. Let $T=\left\{r i: r \in \Re, i^{2}=-1\right\}$, where $\Re$ is the set of all real numbers. Then together with usual ternary multiplication, $T$ forms a ternarysemigroup. Let $r i(\neq 0) \in T$ and $F=\{r i\}$. Then $(r i) F F F y=0$ implies that $y=0$ for all $y \in T$. Hence $T$ is a right stronglyprime ternarysemigroup.

Definition 2.3. Let $A$ be a nonempty subset of a ternarysemigroup $T$. Then the right annihilator of $A$ w.r.t a nonempty subset $B$ of $T$ in $T$, denoted by $r_{s}(A, B)$ is defined by $r_{s}(A, B)=\{x \in T: A B x=\{0\}\}$ [left annihilator, lateral annihilator, annihilator].

Theorem 2.4. A ternarysemigroup $T$ with identity is a right stronglyprime ternarysemigroup if and only if every nonzero ideal of $T$ contains a finitely generated left ideal whose right annihilator w.r.t some finite subset of $T$ is zero.

Proof. Suppose $T$ is a right stronglyprime ternarysemigroup and $I$ is a non zero ideal of $T$. Let $r \neq 0$ be an element of $I$. So $r \in T^{*}$. Now since $T$ is a right stronglyprime ternarysemigroup, corresponding to $r$, there exists a finite subset $F$ of $T$, satisfying $r F F F T=0 \Rightarrow t=0$. Now $r F F \subseteq I$ and $r F F$ is finite. Let $L$ be the left ideal of $T$ generated by $r F F$. i.e $L=\operatorname{TTrFF}$. So $L \subseteq I$. Now let $L F t=0$. As $T$ contains identity, $r F F \subseteq \operatorname{TTrFF\Rightarrow }$ $r F F F t \subseteq T \operatorname{TrFFFt}=L F t=0$, hence $t=0$. Thus $I$ contains the finitely generated left ideal $L$ whose right annihilator w.r.t $F$ is zero.

Conversely, let $x(\neq 0) \in T$. Then $\langle x\rangle$ is a nonzero ideal of $T$. Then by hypothesis, there exists a finite subset $F^{\prime}$ such that right annihilator of the left ideal $L$ generated by $F^{\prime}$ w.r.t finite subset $F$ of $T$ is zero. In other words 
$L F y=\{0\}$ implies that $y=0$, for all $y \in T$. If possible let $x T T=\{0\}$. Then $\langle x\rangle T T=\{0\}$. Since $F^{\prime} F a \subseteq\langle a\rangle=T T a T T \subseteq\langle a\rangle T T=\{0\}$ ( since $T$ contains identity ), we have $F^{\prime} F a=\{0\} \Rightarrow L F x=\{0\}$, as $L=T T F^{\prime}$; but $L F x=\{0\}$ $\Rightarrow x=0$, a contradiction. Thus $x T T \neq 0$. So there exist $r, a \in T$ such that $x r a \neq 0$. Let $I=\langle x r a\rangle, I \neq(0)$.

Then by hypothesis, there exist a finite subset $G^{\prime}$ of $I$ and $H=T T G^{\prime}$ satisfying $H H^{\prime} y=\{0\} \Rightarrow y=0$ for all $y \in T$ where $H^{\prime}$ is a finite subset of $T$.

Let $G^{\prime}=\left\{\bigcup_{p=1}^{t} \xi_{p} \gamma_{p} x r a \lambda_{p} \eta_{p}\right\}$ where $t \in Z^{+}$and $\xi_{p}, \gamma_{p}, \lambda_{p}, \eta_{p} \in T$. Let $F^{\prime \prime}=\left\{a, a \lambda_{p} \eta_{p}\right\} ; p=1,2 \ldots . t$ where $t \in Z^{+}$; then $F^{\prime \prime}$ is a finite subset of $T$. Let $F_{1}=\{r\} ; F_{2}=F^{\prime \prime}$ and $F_{3}=H^{\prime}$. Now $x F_{1} F_{2} F_{3} y=\{0\}, H H^{\prime} y=\{0\} \Rightarrow y=0$. So $T$ is right stronglyprime.

Theorem 2.5. A ternarysemigroup $T$ with identity is right stronglyprime if and only if every nonzero ideal of $T$ contains a finite subset $B$ such that the right annihilator of $B$ w.r.t a finite subset of $T$ is zero.

Proof. Suppose $T$ is right stronglyprime ternarysemigroup and $A$ is any non zero ideal of $T$. Let $x(\neq 0) \in A$. Since $T$ is a right stronglyprime ternarysemigroup, $x$ has a right insulator $F$. Let $B=x F F$, then $B$ is a finite subset of $A$ and right annihilator of $B$ w.r.t $F$ is zero. i.e $r_{s}(B, F)=0$.

Conversely, suppose that every non zero ideal of $T$ contains a finite subset whose right annihilator w.r.t a finite subset $F$ of $T$ is zero. Let $x(\neq 0) \in T$. Then $\langle x\rangle$ is a non zero ideal of $T$. Then there exists finite subsets $F^{\prime}$ of $\langle a\rangle$ and a finite subset $F$ of $T$ such that $F^{\prime} F y=\{0\} \Rightarrow y=0$ for all $y \in T$. Now proceeding as in the proof of the theorem 2.4 we can show that $T$ is right stronglyprime ternarysemigroup.

Proposition 2.6. Every simple ternarysemigroup with identity is a right stronglyprime.

Proof. Let $T$ be a simple ternarysemigroup with identity. Since $T$ admits identity there exist elements $\left\{\left(e_{i}, f_{i}\right) \in T \times T(i=1,2, \ldots n)\right\}$ such that $\bigcup_{i=1}^{n} e_{i} f_{i} x=\bigcup_{i=1}^{n} e_{i} x f_{i}=\bigcup_{i=1}^{n} x e_{i} f_{i}=x$ for all $x \in T$. Since $T$ is simple, $T$ is the only non zero ideal of $T$. Now $G=\left\{e_{i}\right\}(i=1,2, \ldots . n), H=\left\{f_{i}\right\}$ $(i=1,2, \ldots . n)$ are finite subsets of $T$ and right annihilator of $G$ w.r.t $H$ is $\{0\}$, since $G H y=\{0\} \Rightarrow \sum_{i=1}^{n} e_{i} f_{i} y=0 \Rightarrow y=0$, so by theorem 2.5, $T$ is right stronglyprime ternarysemigroup.

Definition 2.7. A class $\rho$ of a ternarysemigroup is called hereditary $A$ is 
an ideal of a ternary semigroup $T$ and $T \in \rho$ implies $A \in \rho$.

Proposition 2.8. The class of all right stronglyprime ternarysemigroups is hereditary.

Proof. Let $T$ be a right stronglyprime ternarysemigroup and $A$ be an ideal of $T$. If $A=(0)$, then $A$ is trivially right stronglyprime ternarysemigroup. So we assume that $A \neq(0)$ and let ' $x$ ' be a non zero element of $A$. Then $x \in T^{*}$. Since $T$ is right stronglyprime ternarysemigroup, there exists a finite subset $F$ of $T$ depending on ' $x$ ' such that $x F F F y=0 \Rightarrow y=0 \rightarrow(1)$.

Obviously $F_{1}=F F F x F F F$ and $F_{2}=\{x\}$ are two finite subsets of $A$. Now suppose That $x F_{1} F_{2} F_{1} y=0$ where $y \in I$. Then $x F F F x F F F x F F F x F F F y=$ 0 . Now by (1) we have $x F F F x F F F x F F F y=0$. Continuing this process we get $y=0$. So $A$ is right stronglyprime ternarysemigroup. Hence the class of all right stronglyprime ternarysemigroups is hereditary.

Definition 2.9. An element $x$ of a ternarysemigroup $T$ is called an idempotent if $x^{3}=x$.

Proposition 2.10. If $T$ is a right stronglyprime ternarysemigroup and ' $i$ is a non zero idempotent element in $T$, then $i T i$ is a right stronglyprime ternarysubsemigroup of $T$.

Proof. Obviously $i T i$ is a ternarysubsemigroup of $T$. Let $i t i$ be a non zero element of $i T i$. Then $i t i \in T$. Since $T$ is right stronglyprime ternarysemigroup, corresponding to iti there exist a right insulator $F=\left\{f_{1}, f_{2}, \ldots f_{k}\right\}$ (say) in $T$. Let $F_{1}=\{i\}, F_{2}=\left\{i f_{r} f_{s} f_{t} i / f_{r}, f_{s}, f_{t} \in\right.$ Fwhere $\left.1 \leq r ; s, t \leq k\right\}$. Consider (isi) $F_{1} F_{2} F_{1}($ iyi $)=0 \Rightarrow($ isi $) i\left(i f_{r} f_{s} f_{t} i\right) i($ iyi $)=0$ where $f_{r}, f_{s}, f_{t} \in F \Rightarrow$ is $\left(\right.$ iii) $f_{r} f_{s} f_{t}($ iii $) y i=0 \Rightarrow($ isi $) f_{r} f_{s} f_{t}($ iyi $)=0 \Rightarrow$ (isi) FFF (iyi) $=0$ which implies $i y i=0$. This $i T i$ is a right stronglyprime ternary subsemigroup of $T$.

Definition 2.11. An ideal $A$ of a ternarysemigroup $T$ is called right stronglyprime ideal of $T$ if for $x \notin A$, there exist a finite subset $F^{\prime}$ of $\langle x\rangle$ and a finite subset $F$ of $T$ such that $F^{\prime} F y \subseteq A \Rightarrow y \in A$.

Theorem 2.12. Every proper right stronglyprime ideal of a ternarysemigroup $T$ is a prime ideal of $T$.

Proof. Suppose $A$ right stronglyprime ideal of $T$. Let $X, Y, Z$ be three ideals of $T$ such that $X Y Z \subseteq A$. Suppose that $X \nsubseteq A$ and $Y \nsubseteq A$. Let $x \in X \backslash A$ and $y \in Y \backslash A$. Since $A$ is a right stronglyprime ideal of $T$ and $X \notin A$, there exists a finite subsets $F^{\prime}$ of $\langle x\rangle$ and a finite subset $F$ of $T$ such that $F^{\prime} F a \subseteq A \Rightarrow a \in A . \rightarrow(1)$ 
Let $y_{i} \in Y, t \in T$ and $z_{i} \in Z$. Now we have $F^{\prime} F y_{i} t z_{i} \subseteq X T Y T Z \subseteq A$. So by (1) $y_{1} t z_{1} \in A$. Thus $Y T Z \subseteq A$. Also $y \notin A$, so there exists a finite subset $F^{\prime \prime}$ of $\langle y\rangle$ and a finite subset $F_{1}$ of $T$ such that $F^{\prime \prime} F_{i} b \subseteq A \rightarrow b \in A . \rightarrow(2)$

Now let $z \in Z$, then $F^{\prime \prime} F_{1} z \subseteq Y T Z \subseteq A \rightarrow z \in A$ by (2). Thus $Z \subseteq A$. Again let $X \nsubseteq A$ and $Z \nsubseteq A$. If possible, let $Y \nsubseteq A$. Then by above argument we have $Z \subseteq A$, a contradiction. So, $Y \subseteq A$. Similarly, if $Z \nsubseteq A$ and $Y \nsubseteq A$, then we have $X \subseteq A$. Thus $X Y Z \subseteq A \Rightarrow X \subseteq A$ or $Y \subseteq A$ or $Z \subseteq A$. So $A$ is a prime ideal of $T$.

Definition 2.13. Right stronglyprime radical of a ternarysemigroup $T$, denoted by $T P(T)$ is defined by

$$
T P(T)=\bigcap\{A: A \text { is a right stronglyprime ideal of } T\} .
$$

Definition 2.14. A non empty subset $G$ of a ternarysemigroup $T$ is called an sp-system if for any $g \in G$ there is a finite subset $F_{1}\langle g\rangle$ and a finite subset $F_{2}$ of $T$ such that $F_{1} F_{2} z \cap G \neq \phi$ for all $z \in G$.

Theorem 2.15. A proper ideal $A$ of a ternarysemigroup $T$ is right stronglyprime if and only if $T \backslash A$ is an sp-system.

Proof. Let $A$ be a right strongly prime ideal of $T$. Let $g \in T \backslash A$. Then $g \notin A$. So there exists a finite subsets $F^{\prime}$ of $\langle g\rangle$ and $F$ of $T$ such that $F^{\prime} F b \subseteq A$ implies that $b \in A$. This implies that $F^{\prime} F z \cap T \backslash A \neq \emptyset$ for all $z \in T \backslash A$. Hence $T \backslash A$ is an sp-system.

Conversely, suppose that $T \backslash A$ is an sp-system. Let $a \notin A$. Then $a \in T \backslash A$. So there exsits a finite subset $F^{\prime}$ of $\langle a\rangle$ and $F$ of $T$ such that $F^{\prime} F z \cap T \backslash A \neq \emptyset$ for all $z \in T \backslash A$. Let $F^{\prime} F b \subseteq A$. Then $F^{\prime} F b \cap(T \backslash A)=\emptyset$. If possible, let $b \notin A$. Then $b \in T \backslash A$ which implies that $F^{\prime} F b \cap(T \backslash A) \neq \emptyset$, a contradiction. So $b \in A$ and hence $A$ is a right strongly prime ideal of $T$.

Definition 2.16. A pair of subsets $(G, I)$ where $I$ is an ideal of a ternarysemigroup $T$ and $G$ is a nonempty subset of $T$ is called a super sp-system of $T$. $G \cap I$ contains no non zero element of $T$ and for any $g \in G$, there exist a finite subset $F$ of $\langle g\rangle$ and a finite subset $F^{\prime}$ of $T$ such that $F F^{\prime} z \cap G \neq \phi$ for all $z \notin I$.

Proposition 2.17. An ideal $A$ of a ternarysemigroup $T$ is a right stronglyprime if and only if $(T \backslash A, A)$ is a super sp-system of $T$.

Proof. Let $A$ be a right stronglyprime ideal of $T$. So $T \backslash A$ is an sp-system by theorem 2.15. Thus for any $y \in T \backslash A$, there exists a finite subset $F$ of $\langle y\rangle$ and a finite subset $F^{\prime}$ of $T$ such that $F F^{\prime} z \cap(T \backslash A) \neq \phi$ for all $z \notin A$. Also 
$(T \backslash A) \cap A$ contains no non zero element of $T$. Thus the pair $(T \backslash A, A)$ is a super sp-system of $T$.

Converse follows from the definition.

Theorem 2.18. For any ternarysemigroup $T, T P(T)=[x \in T /$ whenever $(G, P)$ is a super sp-system for some ideal $P$ of $T$ and $x \in G$, then $0 \in G]$.

Proof. Let $x \in T P(T)$. If possible, let $(G, P)$ be a super sp-system with $x \in G$ and $0 \notin G$. Then $G \cap P=\phi$. By Zorn's lemma, choose an ideal $Q$ with $P \subseteq Q$ and $Q$ is maximal with respect to $G \cap Q=\phi$. We now prove that $Q$ is a right stronglyprime ideal of $T$. Let $a \notin Q$. Then there exist $g \in G$ such that $\langle g\rangle \subseteq Q \cup\langle a\rangle$. Since $(G, P)$ is a super sp-system, there exists a finite subset $F=\left\{f_{1}, f_{2}, \ldots f_{k}\right\} \subseteq\langle g\rangle$ and a finite subset $F^{\prime}$ of $T$ such that $F^{\prime} z \cap G \neq \phi$ for all $z \notin P \rightarrow(1)$.

Since $F \subseteq\langle g\rangle \subseteq Q \cup\langle a\rangle$ each $f_{i}$ is of the form $f_{i}=q_{i} \cup a_{i}$ for some $q_{i} \in Q$ and $a_{i} \in\langle a\rangle$. Let $F^{*}=\left\{a_{1}, a_{2}, \ldots a_{k}\right\}$ then $F \subseteq\langle a\rangle$. Let $z \in T$ be such that $F^{*} F^{\prime} z \subseteq Q$. Now if $z \notin Q$, then $z \notin P$ so from (1) we have $F F^{\prime} z \cap G \neq \phi$; but $f_{i} F^{\prime} z=\left(q_{i} \cup a_{i}\right) F^{\prime} z=q_{i} F^{\prime} z \cup a_{i} F^{\prime} z \subseteq Q \cup F^{*} F^{\prime} z \subseteq Q \cup Q=Q$ for all $i \in\{1,2, \ldots k\}$. So $F F^{\prime} z \subseteq Q$. Hence $G \cap Q=\phi$, a contradiction. Hence $z \in Q$. So $Q$ is a right stronglyprime ideal of $T$. Now as $T P(T) \subseteq Q$. So $x \in Q$. But by assumption $x \in G$, a contradiction. Hence $0 \in G$.

Conversely, suppose $B=[x \in T /$ when ever $(G, P)$ is a super sp-system for some ideal $P$ of $T$ and $x \in G$, then $0 \in G]$. Let $x \in B$. If possible let $x \notin T P(T)$. Then there exist a right stronglyprime ideal $A$ of $T$ such that $x \notin A$. Then $(T \backslash A, A)$ is a super sp-system, where $x \in T \backslash A$ but $0 \notin T \backslash A$, a contradiction. Hence the converse part.

\section{References}

[1] A. Anjaneyulu, Semigroups in which prime ideals are maximal, Semigroup Forum, 22 (1981), 151-158.

[2] T.K. Dutta, M.L. Das, On strongly prime semiring, Bull. Malays. Math. Sci. Soc., 30, No. 2 (2007), 135-141.

[3] F. Kasner, An extension of the group concept, Bull. Amer. Math. Society, 10 (1904), 290-291.

[4] S. Kar, On ideals in ternary semigroups, Int. J. Math. Gon. Sci., 181 (2005), 3015-3023.

[5] S. Kar, J. Sircar, S. Mandal, On right strongly prime ternary semirings, East-West Journal of Mathematics, 12, No. 1 (2010), 59-68.

[6] D.H. Lehmer, A ternary analogue of abelian groups, Amer. Jour. Math., 39 (1932), 329-338. 
[7] Y. Sarala, A. Anjaneyulu, Rao D. Madhusudhana, Ideals in ternary semigroups, International e-Journal of Mathematics and Engineering, 203 (2013), 1950-1968.

[8] F.M. Sioson, Ideal theory in ternary semigroups, Math. Japan, 10 (1965), 63-84.

[9] M. Shabir, S. Bashir, Prime ideals in ternary semigroups, Asian European Journal of Mathematics, 2 (2009), 139-152. 
